All-diamond functional surface micro-electrode arrays for brain-slice neural analysis

Peer-reviewed author version

VAHIDPOUR, Farnoosh; Curley, Lowry; Biro, Istvan; MCDONALD, Matthew; CROUX, Dieter; POBEDINSKAS, Paulius; HAENEN, Ken; Giugliano, Michele; Zivcova, Zuzana VIckova; Kavan, Ladislav \& NESLADEK, Milos (2017) All-diamond functional surface micro-electrode arrays for brain-slice neural analysis. In:

PHYSICA STATUS SOLIDI A-APPLICATIONS AND MATERIALS SCIENCE, 214(2) (Art No 1532347).

DOI: $10.1002 /$ pssa.201532347

Handle: http://hdl.handle.net/1942/24158 


\section{Physica Status Solidi A: Applications and Materials Science All-diamond functional surface micro-electrode arrays for brain-slice neural analysis --Manuscript Draft--}

Manuscript Number:

Full Title:

Article Type:

Section/Category:

Keywords:

Corresponding Author:

Corresponding Author Secondary Information:

Corresponding Author's Institution:

Corresponding Author's Secondary Institution:

First Author:

First Author Secondary Information:

Order of Authors:

Order of Authors Secondary Information:

Abstract:

Additional Information:

Question

Please submit a plain text version of your cover letter here.

Please note, if you are submitting a
pssa.201532347R3

All-diamond functional surface micro-electrode arrays for brain-slice neural analysis

Original Paper

Microelectrode arrays; Surface termination; Impedance spectroscopy

Farnoosh Vahidpour

IMO-IMOMEC, Hasselt University

Diepenbeek, Select State or Province BELGIUM

IMO-IMOMEC, Hasselt University

Farnoosh Vahidpour

Farnoosh Vahidpour

Lowry Curley

István Biró

Matthew McDonald

Dieter Croux

Paulius Pobedinskas

Ken Haenen

Michele Giugliano

Zuzana Vlčková Živcová

Ladislav Kavan

Milos Nesládek

Diamond-based microelectrode arrays were fabricated by using nano crystalline diamond as an insulating layer and conductive boron-doped in order to used them for analysis of brain cortical slices. MEA surface is solely composed of diamond, exposed to the cells. The impedance measurements showed negligible cross-talk between neighbouring diamond microelectrodes. Local field potentials related to neural signals were then successfully recorded from pharmacologically disinhibited rat cortical tissue slices, mechanically coupled on the surface of the MEA. The noise level of the diamond MEAs was found to be lower than commercial Pt-based MEAs, under identical measurement conditions.

\section{Response}

Dear Editorial Office, Physica Status Solidi A;

We have the pleasure resubmitting a new version of our manuscript "All-diamond functional surface micro-electrode arrays for brain-slice neural analysis". 
revision of your manuscript, there is an opportunity for you to provide your responses to the reviewers later; please do not add them to the cover letter.
We would like to thank the referee for their valuable comments and input.

With the best regards,

Farnoosh Vahidpour 


\title{
All-diamond functional surface micro-electrode arrays for brain-slice neural analysis
}

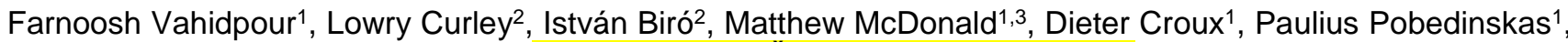 \\ Ken Haenen ${ }^{1,3}$, Michele Giugliano ${ }^{2-5}$, Zuzana Vlčková Živcová6, Ladislav Kavan ${ }^{6}$, Milos Nesládek¹,3 \\ ${ }^{1}$ Institute for Materials Research (IMO), Hasselt University, Belgium \\ ${ }^{2}$ Theoretical Neurobiology \& Neuroengineering Laboratory, Dept. Biomedical Sciences, Universiteit Antwerpen, Belgium \\ ${ }^{3}$ IMOMEC, IMEC vzw, Belgium \\ ${ }^{4}$ Brain Mind Institute, Swiss Federal Institute of Technology of Lausanne, Switzerland \\ ${ }^{5}$ Department of Computer Science, University of Sheffield, United Kingdom \\ ${ }^{6}$ Department of Electrochemical Materials, J. Heyrovsky Institute of Physical Chemistry of the AS CR, v.v.i., Czech Republic
}

Received ZZZ, revised ZZZ, accepted ZZZ

Published online ZZZ (Dates will be provided by the publisher.)

Keywords Microelectrode arrays, Surface termination, Impedance spectroscopy

Corresponding author: e-mail: farnoosh.vahidpour@uhasselt.be

\begin{abstract}
Diamond-based microelectrode arrays were fabricated by using nano crystalline diamond as an insulating layer and conductive boron-doped in order to used them for analysis of brain cortical slices. MEA surface is solely composed of diamond, exposed to the cells. The impedance measurements showed negligible cross-talk between neighbouring diamond microelectrodes. Local field po-
\end{abstract}

1 Introduction For over a decade, academic and industrial laboratories have worked on improving the technology for electrophysiological interfacing of neural tissue to substrate-integrated microelectrode arrays (MEAs). MEAs essentially serve as neural interfaces that connect the neurons to the external measurement circuit to detect electrical potential associated with the function of excitable cells. MEAs are nowadays employed both in vivo and in vitro, used, for example, for brain research or various neuropharmacological applications ${ }^{1}$. For each of specific applications, the geometry, shape, and the material of the MEAs play critical roles. To date, MEAs are fabricated by employing a variety of conducting materials such as titanium nitride $(\mathrm{TiN})$, iridium oxide ( $\mathrm{IrO})$, platinum $(\mathrm{Pt})$, gold $(\mathrm{Au})$, indium tin oxide (ITO), silicon ( $\mathrm{Si}$ ) and titanium $(\mathrm{Ti})^{2-8}$ as the electrode material. However, the electrode material stability in biologic environment is an important issue. In particular, upon electrical extracellular stimulation, the mi- tentials related to neural signals were then successfully recorded from pharmacologically disinhibited rat cortical tissue slices, mechanically coupled on the surface of the MEA. The noise level of the diamond MEAs was found to be lower than commercial Pt-based MEAs, under identical measurement conditions.

Copyright line will be provided by the publisher

croelectrode and interface characteristic in the contact with the tissue can change, altering the MEA response ${ }^{3,9-11}$. Most of the MEAs are formed by using a combination of different materials for electrodes and the insolating surface (such as $\mathrm{SiN}_{\mathrm{SiO}}$, polymers etc.), with distinctive characteristics for interaction with neural cells.

Artificial man-made, Chemical Vapour Deposition (CVD) diamond is one of very interesting thin film materials for the MEA fabrication due to its biological inertness, chemical stability and availability of both highly insolating and highly conducting B-doped thin diamond films. It has been widely reported that nano crystalline diamond (NCD) is biocompatible as deduced from both in in vitro ${ }^{12,13}$ and in vivo $^{14,15}$ studies, exploring imaging, drug delivery, diagnostic or treatments contexts ${ }^{10,16-18}$. Additionally diamond also exhibits reduced bio-fouling. Boron doped NCD (BNCD) is electrically conductive with a wide electrochemical potential window ${ }^{19}$. These properties make dia- 
mond an attractive platform for fabrication of active bioelectronic devices ${ }^{11,20-22}$. Thus, one immediate application of BNCD are MEAs for in vitro electrophysiological studies and pharmacological screening in neuroscience. The aim of this study is two - fold. We prepare MEAs in which the cells are exposed only to diamond surface, by using both metallically conductive as well as isolating diamond thin films for MEAs, providing thus homogenous surface for interaction with neural cultures. We study the interaction of both the conductive and isolating diamond surfaces with cellular environment and demonstrate the MEA functionality using acute brain slices. The thin flat diamond MEA with microelectrode channels was used to monitor local responses of single neurons or populations of cells.

\section{Experimental methods}

2.1 Substrate preparation $49 \mathrm{~mm}$ by $49 \mathrm{~mm}$ fused silica substrates were employed for the fabrication of the MEAs Fused silica has a relatively high melting point and it is stable even at high temperatures used for the diamond growth $\left(600-800^{\circ} \mathrm{C}\right)$. Another benefit of fused silica for constructing MEAs is its optical transparency, allowing optical simultaneous imaging in transmission microscopy. Before the diamond growth, fused silica substrates were cleaned according to the standard wafer cleaning procedure RCA 1 and RCA $2^{23}$ [Figure 3, step\#1]. To initiate diamond growth by plasma enhanced microwave chemical vapour deposition (PE MW CVD), diamond nuclei have to be provided on the substrate surface. There are various approaches for such surface pre-treatment. One is the seeding approach with diamond nano particles by submerging the substrate into a water based particle colloid. The other is abrading the surface of the substrate using diamond powder, leaving diamond residues on the surface ${ }^{11}$. In this study, we have applied the former approach ${ }^{24}$. A colloid of nano diamonds (NanoAmando®B from NanoCarbon Research Institute Ltd., Nagano, Japan) has been used. These detonation nano diamonds had the average diameter of $5 \mathrm{~nm}$. The $\mathrm{Z}$ potential of the particles was $\sim 50 \mathrm{mV}$ and the surface was predominantly $\mathrm{sp} 2$ terminated ${ }^{25}$. The nano diamond colloid was ultra-sonicated prior to the seeding, to break up any large clusters of nano diamond particles to produce mono dispersion as confirmed by dynamic light scattering (DLS). The cleaned silica substrates were immersed into this colloid for a minute. Then, the samples were spun for 40 seconds until dry, using a spin coater. The seeded substrates were flushed with Milli-Q water for the first 10 seconds to remove residual nano diamonds from the surface, leaving a nano diamond monolayer.

\subsection{Fabrication steps of microelectrode arrays To} fabricate diamond MEAs, diamond layers were grown on the seeded substrates. 150nm thick films of BNCD (as the conductive layer) were grown first using an ASTeX 6550 series using MW-PECVD system [Figure 3, step\#2]. 375 sccm of $\mathrm{H}_{2}, 25 \mathrm{sccm}(5 \%)$ Methane and $100 \mathrm{sccm}$ (8000ppm) of Trimethylboron (TMB) were used as gas mixture for the MW-PECVD growth. TMB was used in Hydrogen mixture. $8000 \mathrm{ppm}$ TMB leads to the boron concentration of about $3 \mathrm{E} 21 \mathrm{~cm}^{-3}$. The substrate temperature of $600{ }^{\circ} \mathrm{C}$ was used for the diamond growth, selected to obtain relatively smooth surface whilst keeping sufficiently high boron incorporation. The substrate temperature was controlled using a Williamson Pro 92-38 infrared pyrometer. The growth conditions were optimized to achieve a high $\mathrm{sp}^{3}$ carbon purity ${ }^{19}$ and high B-doping level $\left(>10^{20} \mathrm{~cm}^{-3}\right.$ ) leading to a sheet resistance (of about $500 \Omega /$ square) of the film grown under the conditions: pressure 22 Torr and MW power 3500 Watt. Once the BNCD film growth was completed, a photolithography step was performed [Figure 3, step\#3] using negative photoresist NR9-3000PY (Futurrex, inc.) and a photolithography mask, as shown in Figure 1.

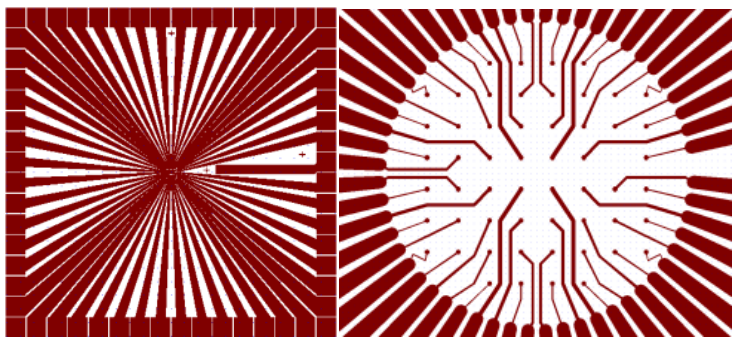

Figure 1: Left: Design of the Lithography mask for conductive areas. Right: The zoomed center of the mask showing the channels

Subsequently, a thin metal layer was deposited on the surface for negative lift-off lithographic processing and used as a mask for definition of electrodes [Figure 3, step\#4]. For the purpose of masking, different metals were studied. Because subsequently an un-doped diamond layer is grown on top of patterned metallic films, one has to be cautious in the metal selection to achieve optimal adhesion to diamond. In case of chromium, the adhesion of diamond to $\mathrm{Cr}$ was not sufficient. Titanium made a good adhesion to diamond, but it was not suitable for the wet HF etching that attacks the quartz substrate. Therefore, tungsten was used as a protection mask, exhibiting a good adhesion to diamond, also resisted the diamond growth process, and the final etching step (by $\mathrm{H}_{2} \mathrm{O}_{2}, 30 \%$ ) is not harmful for the substrate. Tungsten has a relatively low resistivity: $10^{-6} \Omega \mathrm{cm}$ (compared to $\sim 10^{-2} \Omega \mathrm{cm}$ for BNCD) and can be therefore used for enhancing the conductivity on top of BNCD layer, sandwiched between BNCD and NCD. Our experiences indicate only $\sim 20 \mathrm{~nm}$ of $\mathrm{W}$ is sufficient to achieve good masking. Tungsten is deposited by magnetron sputtering using a tungsten target, $50 \mathrm{sccm}$ of argon and 100 Watts power under working pressure of few milli Torr. Further, an acetone-assisted lift off of the metal was carried out to define the conductive microelectrodes (where cells are later exposed to BNCD surface for recording or stimulation), the conductive tracks and the connection pads [see Figure 1, 
also Figure 3, step\#5]. The BNCD film with the mask was exposed to reactive ion etching (RIE) using $\mathrm{O}_{2}$ plasma under the conditions of 300 Watt, $30 \mathrm{sccm} \mathrm{O} \mathrm{O}_{2}$ and a working pressure of a few $\mathrm{m}$ Torr to etch the BNCD layer outside the intended electrode area [Figure 3,step\#6]. Next, a $120 \mathrm{~nm}$ thick NCD film was grown on top of the structure as an insulating layer on the MEA structure [Figure 3, step\#7]. The tungsten layer was kept on the BNCD surface and used later on as an etch-stop layer to protect the BNCD in diamond etching steps. The growth conditions for the NCD film were $495 \mathrm{sccm}$ of $\mathrm{H}_{2}, 5 \mathrm{sccm}(1 \%)$ of methane and 4000 Watt power at a gas pressure of 20 Torr. NCD layer was grown in a temperature between 650 to $700{ }^{\circ} \mathrm{C}$. After NCD growth, another step of photolithography and metal mask (chromium) deposition was applied [Figure 3, step\#8] using the insulating photolithographic mask [Figure 2] to mask the insulating areas, subsequently remove the NCD on microelectrodes and to open NCD by RIE down to BNCD [Figure 3, steps\# 9,10,11]. The chromium layer was deposited by applying argon plasma and a chromium target. 100 Watts of power and $50 \mathrm{sccm}$ argon was used under the working pressure of few $m$ Torr to coat the NCD surface.
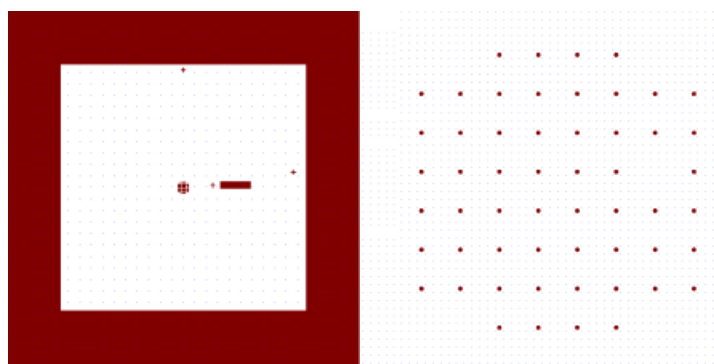

Figure 2: Left: Design of the Lithography mask for NCD (insulating) areas. Right: The zoomed centre of the mask showing channels with $20 \mu \mathrm{m}$ diameter and $200 \mu \mathrm{m}$ spacing.

After RIE processing, chromium mask was removed by wet etching, by using standard $\mathrm{Cr}$ etchant (Sigma Aldrich, Belgium) [Figure 3, step\#12]. Finally, tungsten layer was etched by $\mathrm{H}_{2} \mathrm{O}_{2}$ solution (30\%, Sigma Aldrich, Belgium) [Figure 3, step\#13]. Once the metallic masks were removed, the diamond microelectrode array was produced according to the plan shown in Figure 3. The surfaces available for interaction with neurons remained entirely made of diamond.

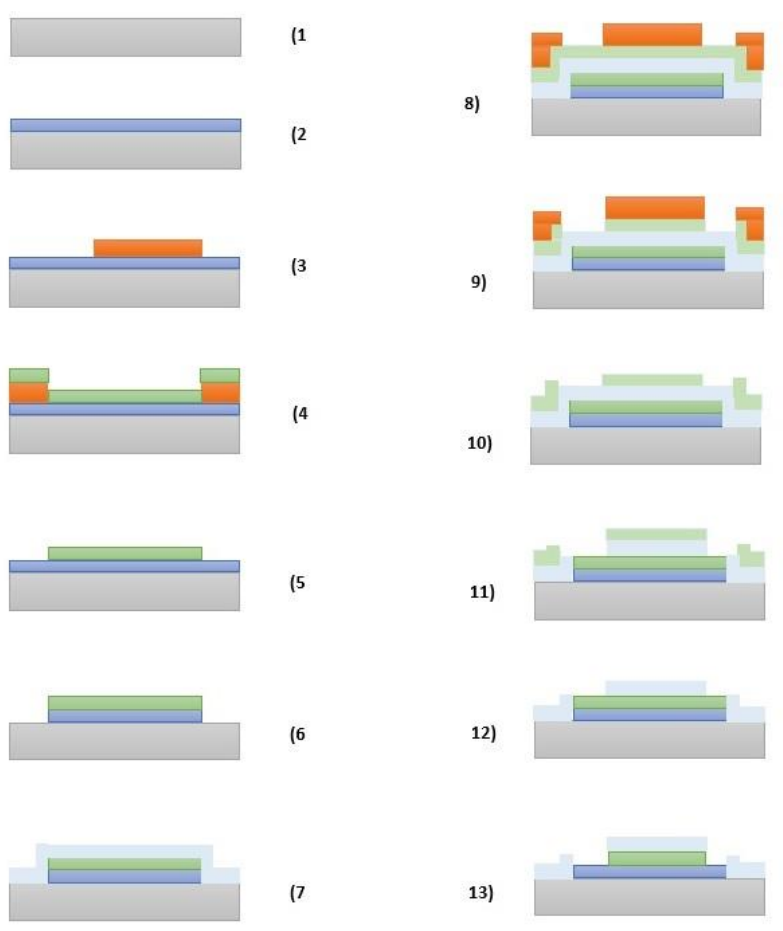

Figure 3: Schematic of complete plan steps for fabrication of diamond MEA:1)Fused Silica wafer, 2) BNCD growth, 3)Photolithography, 4) Metal-1 deposition, 5)Lift off, 6)BNCD etching, 7)NCD grown, 8)Metal-2 deposition and Photolithography, 9)Metal-2 mask etching, 10)Photoresist acetone-cleaning, 11)NCD etching, 12)Metal-2 mask removal, 13)Metal-1 mask removal.

\subsection{Surface termination and wettability of dia- mond}

To study the wettability of both conductive and isolating diamond surfaces in cell experiments, two sets of fused silica substrates were prepared on which NCD and BNCD thin films were subsequently grown. One set of the NCD and BNCD coated substrates were hydrogen terminated; using hydrogen plasma exposure in the ASTeX reactor $\left(800^{\circ} \mathrm{C} .10\right.$ minutes, cooling in hydrogen). For the other set of substrates, the surfaces were oxygen terminated using UV induced ozone treatment in a PSD series digital UVozone system (Novascan Technologies, Inc.) for 30 minutes to create a homogeneously oxidized surface. The surface wettability by measuring the water droplet contact using OCA15EC Video Based Optical Contact angle Measurement Instrument.

To study the impact of the biological environment on the diamond surface wettability, the same set of samples were later treated with 1:1 Dulbecco's Modified Eagle's Medium (DMEM) and nutrient mixture F-12 from Sigma (Taufkirchen, Germany) containing 10\% fetal bovine serum (FBS), and $1 \%$ penicillin/streptomycin. The changes in diamond surface wettability due to physisorption of molecular or 
ionic species from the cell medium were monitored using the same setup.

To investigate this effect, the incubation was carried out and for a short (1-2 hours) and longer period of time (19 hours) in medium with FBS. After washing the surface in Milli-Q water, the contact angles were re-measured and compared.

\subsection{Electrical characterization of diamond elec- trodes}

The impedance spectroscopy was employed to characterize the diamond MEA and to evaluate the cross talk between the electrodes in dry condition (i.e. through the diamond layers), which is important to be minimized. For cross talk measurement, HP 4194a Impedance/Gain-Phase analyser from Keysight technologies was employed. The impedance for the cross talk evaluation was measured between two microelectrodes using a $50 \mathrm{mV}$ peak-to-peak excitation signal which was applied by the signal generator. The impedance was assessed in two conditions: wet (i.e., in phosphate-buffered saline, PBS) and dry.
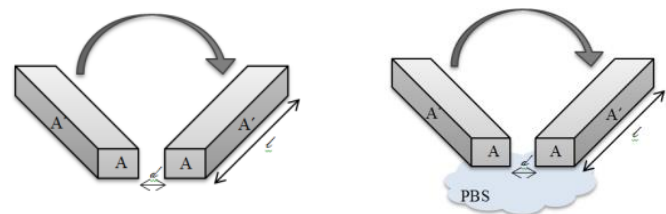

Figure 4: Schematic of the electrodes used in impedance measurements for cross talk evaluation in, left: dry condition and, right: wet condition (in PBS).

For impedance measurement of the electrodes, Solartron 1260 Impedance/Gain-Phase analyser was employed. The same setup was used to evaluate the signal to noise ratio of the MEA. An input AC voltage of $3 \mathrm{~V}$ was applied and the output voltage was measured across the frequency range of $1 \mathrm{MHz}$ to $1 \mathrm{~Hz}$.

\subsection{Brain tissue slices preparation and MEA elec- trophysiology}

Tissue preparation was performed by standard methods ${ }^{26}$, closely following the guidelines of the Ethics Committee of the University of Antwerp. Briefly, 21 days old Wistar rats were anaesthetized with Isoflurane (IsoFlo, Abbott, USA), decapitated, and their brains excised. $300 \mu \mathrm{m}$ thick tissue slices (parasagittal) of the somatosensory cortex were cut by a vibratome (VT1000 S, Leica Microsystems, Diegem, Belgium) in ice-cold Artificial Cerebro-Spinal Fluid (ACSF). The ACSF contained (in mM) $125 \mathrm{NaCl}, 25$ $\mathrm{NaHCO}_{3}, 2.5 \mathrm{KCl}, 1.25 \mathrm{NaH}_{2} \mathrm{PO}_{4}, 2 \mathrm{CaCl}_{2}, 1 \mathrm{MgCl}_{2}, 25$ glucose, balanced by $95 \% \mathrm{O}_{2}, 5 \% \mathrm{CO}_{2}$ and adjusted to $\mathrm{pH}$ 7.3. The same solution was also employed after cut, to incubate slices at $36^{\circ} \mathrm{C}$ for at least $45 \mathrm{~min}$, during slice storage at room temperature, as well as during the electrophysiological recordings, performed at room temperature. All chemicals were obtained from Sigma-Aldrich (Diegem, Belgium).

Slices were trimmed, to a width of $\sim 5-6 \mathrm{~mm}$, and gently placed over the active surface of the diamond MEAs, upon previous treatment with cellulose nitrate (Protran, Fisher Scientific, Belgium; $0.14 \mathrm{mg} / \mathrm{ml}$ in $100 \%$ Methanol). MEAs replaced the chamber of an upright microscope. MEA microelectrodes were then employed to monitor noninvasively the extracellular electrical field of neuronal microcircuits in proximity with the substrate. An ad hoc commercial amplifier was employed - 1060BC, Multichannel Systems GmbH, Reutlingen (Germany) to acquire spontaneous electrical activity at $25 \mathrm{kHz} /$ channel, after 1200x amplification. MC Rack software (Multichannel Systems, Reutlingen, Germany) was employed for disk storage of the digitized data. An acquisition computer with a PCI A/D board (MC Card, 64 channels A/D, 4 DIO, 16bits Multichannel Systems, Reutlingen, Germany), was employed in this experiment.

\section{Results and discussion}

\subsection{Surface characterization of MEAs}

Figure 5 shows the images of functional diamond MEAs fabricated on fused silica substrates. Microscope and SEM images confirmed the successful patterning of diamond and presence of diamond on the well-defined active sites.
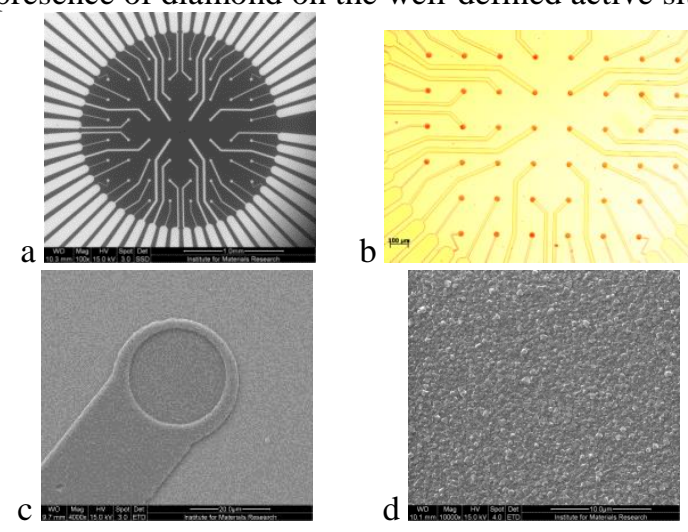

Figure 5: a, b) The central part of the fabricated all diamond MEA. c) One recording channel. d) SEM image of BNCD film

To prepare the MEA for measurement in PBS, a ring was attached to the MEA to function as the PBS holder [Figure 6].

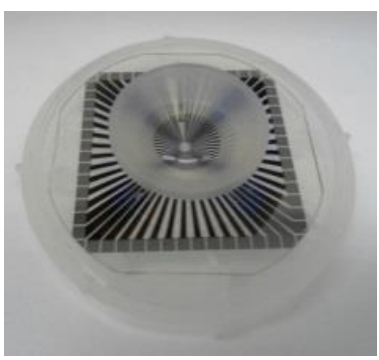

Figure 6: Flexible reusable cell culture silicone chamber (Greiner BioOne) were attached to MEA, creating a bath chamber required for electrochemical impedance measurement under PBS. 


\subsection{Surface termination and wettability of dia-} mond The contact angle measurements showed that the Oxygen terminated diamond surfaces (BNCD and NCD) yielded the contact angle of $20^{\circ}-30^{\circ}$ which is lower than that of hydrogen terminated diamond surfaces (BNCD and NCD) with larger contact angles $\sim 80^{\circ}$, representing hydrophilic and hydrophobic surfaces, respectively. This confirms the known effect that the wettability of the surface increases by oxygen treating the diamond ${ }^{27,28}$.

The contact angles of the substrates after treatment with medium with $10 \%$ FBS changed dramatically [Figure 7]. For the H-treated, originally hydrophobic samples, the contact angle decreased to $60-65^{\circ}$ and for the O-treated, originally hydrophilic, samples, it increased to $55-60^{\circ}$ after medium treatment. The results indicated that the wettability of the surfaces is significantly modified by the interaction of diamond surface with the cell medium containing $10 \%$ FBS. Since the surface of hydrogen or oxygen terminated diamond exhibits different surface band bending respectively ${ }^{29}$, the electrical charge close to the diamond surface can be compensated by ionic species or biomolecules such as protein cocktail leading to wettability change. Interestingly, we see identical change of contact angle for both BNCD and NCD (one would aspects lower band bending for BNCD due to high acceptor concentration of $>10^{20} \mathrm{~cm}^{-}$ ${ }^{3}$ ). This confirms that BNCD and NCD surfaces interact by the same way with the cellular cultures and NCD and BNCD provide a homogeneous interface to neural cultures.

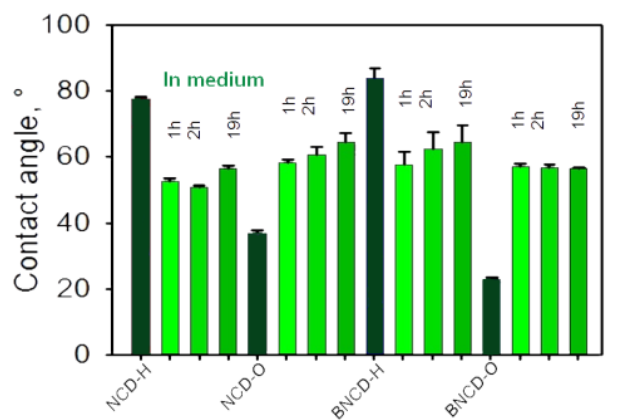

Figure 7: Contact angle measurement across differently terminated diamond surfaces, before and after (1-19h) incubation in medium with FBS.

3.3 Electrical characterization of diamond MEA Electrochemical impedance spectroscopy was used to characterize the conductive diamond MEAs.

Impedance cross-talk data were plotted for a representative pair of conducting electrode lines [Figure 8]. Impedance of the diamond electrodes is measured in dry and in PBS buffer in a frequency range of $1 \mathrm{MHz}$ to $100 \mathrm{~Hz}$.

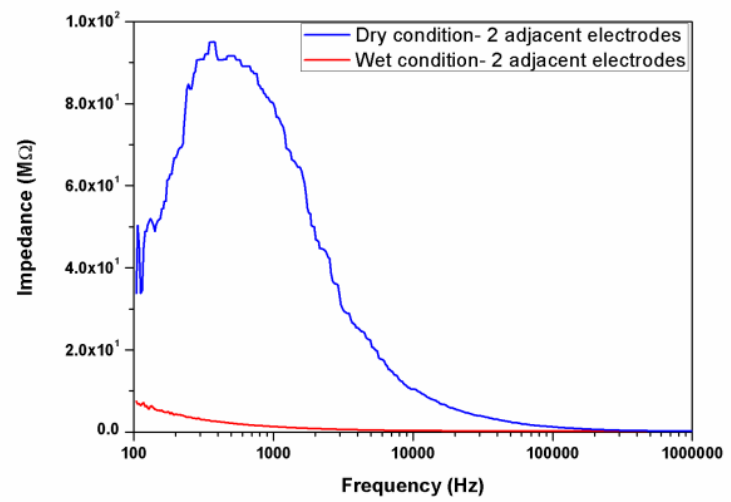

Figure 8: Cross-talk measurement, comparing dry and wet conditions

The cross talk in dry condition (without PBS) shows high impedance. The capacitive and resistive cross talk impedance through the diamond MEA (dry condition) is $\sim 10^{6}$ Ohms. In case of measurement in PBS, the impedance between two adjacent electrodes is lowered due to conduction between the adjacent electrodes through the electrolyte. When neurons are cultured on the MEA or brain slices are placed on the electrodes the impedance to the electrolyte will increase due to sealing the active electrode surface by neurons.

The electrochemical impedance spectrum measured in PBS buffer was fitted using equivalent circuit on Figure 9. In this circuit the Ohmic resistance $R_{\mathrm{s}}$ of the electrolyte solution, electrodes, contacts etc. is in series with the parallel combination of the space charge capacitance (BNCD/electrolyte interface) represented by constant phase element $(C P E)$ and its associated resistance $\left(R_{1}\right)$ in series with diffusion impedance $Z_{\mathrm{w}}$, the so-called Warburg element ${ }^{30}$. This equivalent circuit with diffusion controlled process provided the best fit to our experimental spectrum.

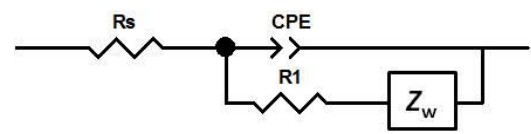

Figure 9: Equivalent circuit used to fit the electrochemical impedance spectrum for diamond-based microelectrode array (MEA), where $R_{\mathrm{s}}$ is Ohmic serial resistance, CPE is constant phase element, $R_{1}$ is the associated charge transfer resistance and $Z_{\mathrm{w}}$ is the Warburg element.

The Nyquist plot of diamond-based microelectrode measured in PBS solution [Figure 10] shows at high frequencies a semicircle corresponds to the electron transfer-limited process followed by a straight line at the lower frequencies range represents the diffusion limited electro-chemical process $^{31}$. 


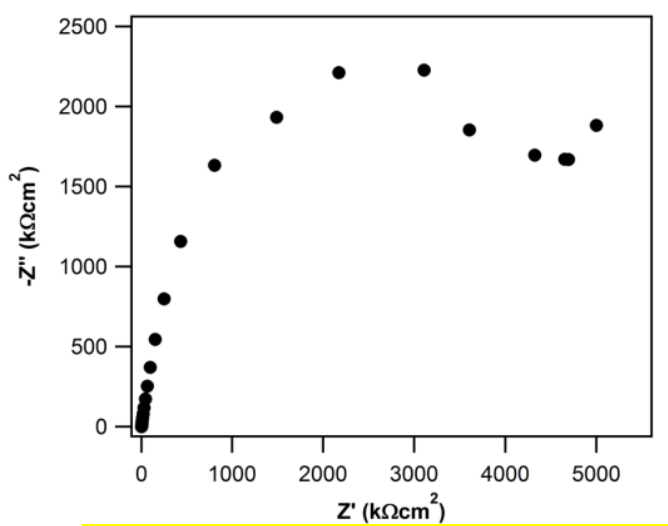

Figure 10: Nyquist plot from electrochemical impedance spectra of diamond-based microelectrode array (MEA) measured at 50 $\mathrm{mV}$ in PBS electrolyte solution. The spectrum was fitted to the equivalent circuit shown in Figure 9.

The impedance of the microelectrodes in PBS was measured as plotted in Figure 11 (Bode plots; $|\mathrm{Z}|$ and phase vs. frequency). The typical electrode showed an impedance of around $180 \mathrm{~K} \Omega$ at $1 \mathrm{kHz}$. This value is comparable to commercial MEAs ( 100-200 kOhms/ $1 \mathrm{kHz})$ and about factor 10 lower than the impedance for the cross talk in wet conditions.
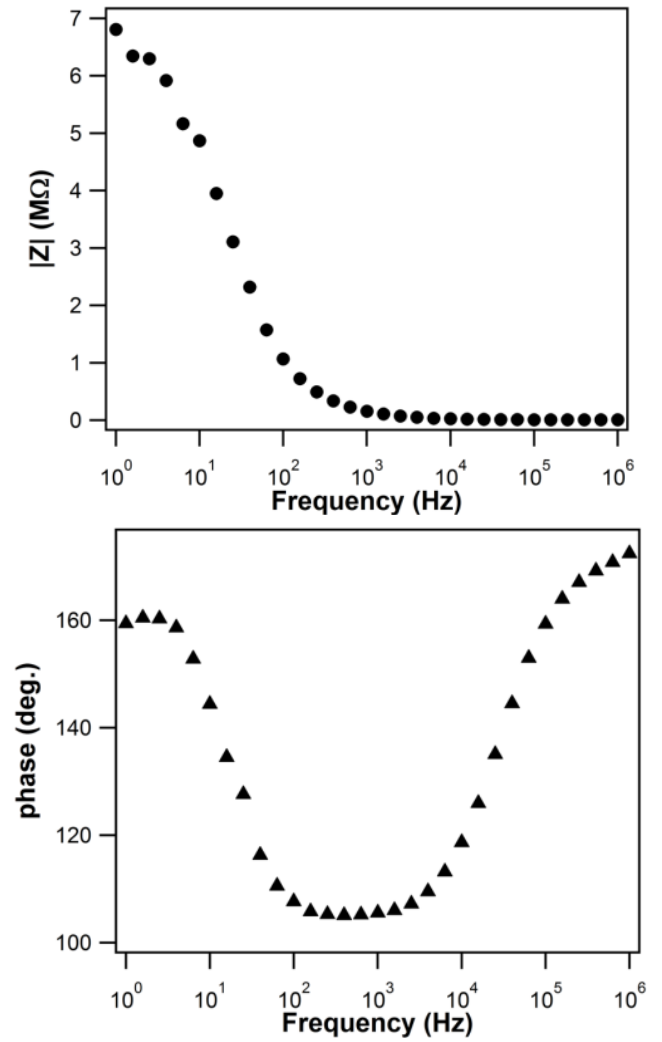

Figure 11: Impedance and phase - frequency diagrams of an example diamond-based microelectrode (circular channels of $20 \mu \mathrm{m}$ diameter)

\subsection{Acute brain slice experiment on diamond} MEAs The diamond MEAs were further used for standard acute brain slice electrophysiological experiments as described in the Methods, under "submerged" conditions. Pharmacological disinhibition was then obtained by bathapplying $20 \mu \mathrm{M}$ of GABAzine (i.e., SR-95531) in ACSF, as a competitive antagonist of $\mathrm{GABA}_{\mathrm{A}}$ receptors. This resulted in spontaneous episodic electrical (epileptiform) activity in vitro.

An A/D converter and a 60 channels analogue amplifier are used for recording the local electrical field potentials, corresponding to the excitable activity of neighbouring neurons. Raw electrical voltages were recorded under a monopolar configuration, i.e., with reference to a synthesized chlorinated silver pellet electrode, immersed in the bath chamber. Waveforms were acquired and plotted using MATLAB, showing Local Field Potentials during an epileptiform epoch [Figure 12], reflecting the synchronous activation of local subset of pyramidal neurons, spatially summated in close proximity of the MEA microelectrodes. Pharmacological disinhibition was necessary as somatosensory cortical slices display very low spontaneous activity.

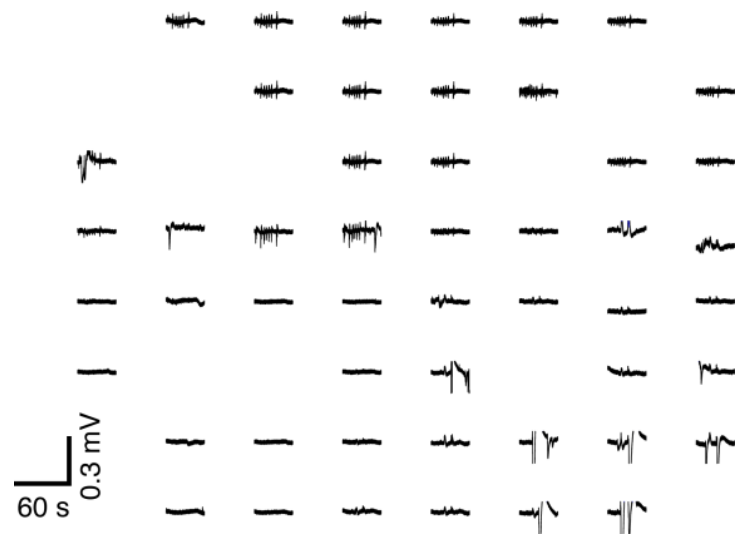

Figure 12: 50 microelectrodes, displayed simultaneously over $1 \mathrm{~min}$ of recording, as a spatial map of the electrical activity of $\sim 100 \mu \mathrm{V}$ across cortical layers

The overall level of background signal associated to the diamond MEAs when emerged to the Artificial CerebroSpinal Fluid (ACSF) without the brain slice has been measured by the same recording setup, under the identical conditions using the 1060BC Multichannel Systems GmbH, Reutlingen (Germany). This background signal recorded from all the channels at $1 \mathrm{kHz}$ is shown in Figure 13. This background signal is composed of the noise of the amplifier alone (also plotted in Figure 13) and the system noise that is composed of noise due to the exposure of MEA to electrochemical environment (impedance is measured with respect to the ground electrode immersed in the electrolyte) at $1 \mathrm{kHz}$. In general the noise was found to be generally lower for diamond microelectrodes, compared to the 
commercial Pt-microelectrodes MEAs (from QWANE Bioscience, Lausanne, Switzerland).

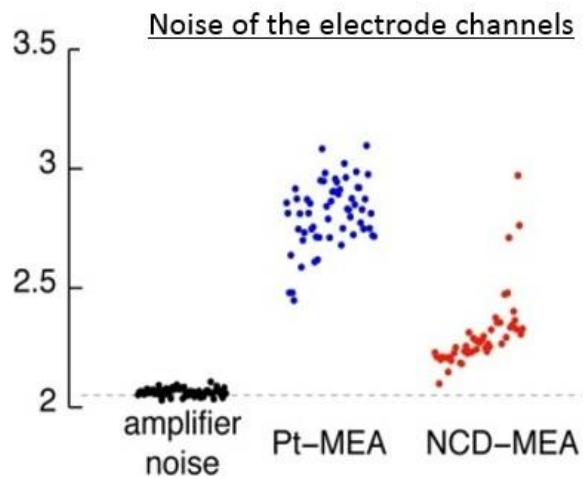

Figure 13: Quantification of the background electrochemical noise at the electrochemical interface between the NCD microelectrodes and the electrolyte (ACSF), compared to control Ptmicroelectrodes of commercial MEA, and to the intrinsic noise of the electronic amplifier.

4 Conclusions Diamond arrays of microelectrodes were successfully fabricated. The diamond MEAs have a single monolithic surface composed only of diamond. The wettability of BNCD and NCD differently terminated diamond surfaces was examined. The treated BNCD and NCD surfaces when exposed to a medium with FBS, showed a change in hydrophobicity, regardless of the prior oxygen or hydrogen treatment. The electrochemical impedance spectroscopy of diamond microelectrodes was analysed and the signal cross-talk in dry conditions between the neighbouring microelectrodes was found negligible. The diamond MEAs allowed detection of the local field potentials for acute rat brain slices, with an overall noise level of the diamond MEA $(\sim 2.3 \mu \mathrm{V}$ at $1000 \mathrm{~Hz})$ generally lower than conventional MEAs $(\sim 2.8 \mu \mathrm{V}$ at $1000 \mathrm{~Hz})$. This all indicates that diamond MEAs have the potential to represent an advanced technology for long term electrical interfacing with neuronal systems.

Acknowledgements We are grateful to M. Wijnants for technical assistance. This work was financially supported by ECFP7 projects (n. 280778-2 NMP-MERIDIAN and n. 264872, ITN-NAMASEN), by the -Research Foundation - Flanders (FWO, grant n. G088812N), by the Belgian Science Policy (grant IAP-P7/20), by the Grant Agency of the Czech Republic (contract No. 13-31783S) and by Hasselt University (BOF). PP is a Postdoctoral Fellow of the Research Foundation - Flanders (FWO).

\section{References}

1. Schurr, A. Editor's Column. i, (1981).

2. Hai, A., Shappir, J. \& Spira, M. E. In-cell recordings by extracellular microelectrodes. Nat. Methods 7, 200-2 (2010).
3.

Fromherz, P. Three levels of neuroelectronic interfacing: silicon chips with ion channels, nerve cells, and brain tissue. Ann. N. Y. Acad. Sci. 1093, 143-60 (2006).

4. Kensall, D. \& James, B. Integrated-Circuit Microelectrodes. 238-247 (1970).

5. Rutten, W. L. C. Selective electrical interfaces with the nervous system. Annu. Rev. Biomed. Eng. 4, 407-52 (2002).

6. MEA-IT Manual.

7. Rizzo, J. F. Methods and Perceptual Thresholds for Short-Term Electrical Stimulation of Human Retina with Microelectrode Arrays. Invest. Ophthalmol. Vis. Sci. 44, 5355-5361 (2003).

8. Weiland, J. D., Anderson, D. J. \& Humayun, M. S. In vitro electrical properties for iridium oxide versus titanium nitride stimulating electrodes. IEEE Trans. Biomed. Eng. 49, 1574-1579 (2002).

9. Spira, M. E. \& Hai, A. Multi-electrode array technologies for neuroscience and cardiology. Nat. Nanotechnol. 8, 83-94 (2013).

10. Grill, A. Diamond-like carbon coatings as biocompatible materials - an overview. 12, 166-170 (2003).

11. Kraft, A., Gmbh, G. \& Str, K. Doped Diamond: A Compact Review on a New, Versatile Electrode Material. 2, 355-385 (2007).

12. Ariano, P. et al. A diamond-based biosensor for the recording of neuronal activity. Biosens. Bioelectron. 24, 2046-50 (2009).

13. Maybeck, V. et al. Boron-doped nanocrystalline diamond microelectrode arrays monitor cardiac action potentials. Adv. Healthc. Mater. 3, 283-289 (2014).

14. Halpern, J. M., Cullins, M. J., Chiel, H. J. \& Martin, H. B. Chronic in vivo nerve electrical recordings of Aplysia californica using a boron-doped polycrystalline diamond electrode. Diam. Relat. Mater. 19, 178-181 (2010).

15. Bergonzo, P. et al. 3D shaped mechanically flexible diamond microelectrode arrays for eye implant applications: The MEDINAS project. Irbm 32, 91-94 (2011).

16. Lin, Y.-C., Perevedentseva, E., Tsai, L.-W., Wu, K.-T. \& Cheng, C.-L. Nanodiamond for intracellular imaging in the microorganisms in vivo. J. Biophotonics 5, 83847 (2012).

17. Allen, M. \& Rushton, N. Diamond-like. 6605, (1994).

18. Tang, L., Tsai, C., Gerberich, W. W., Kruckebeu, L. \& Kania, D. R. Biocompatibility of chemical-vapourdeposited diamond. 16, 483-488 (1995).

19. Živcová, Z. V. et al. Electrochemistry and in situ Raman spectroelectrochemistry of low and high quality boron doped diamond layers in aqueous electrolyte solution. Electrochim. Acta 87, 518-525 (2013). 
20. Nemanich, R. J., Carlisle, J. a., Hirata, A. \& Haenen, K. CVD diamond-Research, applications, and challenges. MRS Bull. 39, 490-494 (2014).

21. Halpern, J. M. et al. Diamond electrodes for neurodynamic studies in Aplysia californica. Diam. Relat. Mater. 15, 183-187 (2006).

22. Dankerl, M. et al. Diamond Transistor Array for Extracellular Recording From Electrogenic Cells. $A d v$. Funct. Mater. 19, 2915-2923 (2009).

23. Kern, W. The Evolution of Silicon Wafer Cleaning Technology. J. Electrochem. Soc. 137, 1887 (1990).

24. Pobedinskas, P. et al. Surface plasma pretreatment for enhanced diamond nucleation on AlN. Appl. Phys. Lett. 102, 201609 (2013).

25. Williams, O. a. et al. Size-dependent reactivity of diamond nanoparticles. ACS Nano 4, 4824-4830 (2010).

26. Köndgen, H. et al. The dynamical response properties of neocortical neurons to temporally modulated noisy inputs in vitro. Cereb. Cortex 18, 2086-2097 (2008).

27. Krueger, A. \& Lang, D. Functionality is key: Recent progress in the surface modification of nanodiamond. Adv. Funct. Mater. 22, 890-906 (2012).

28. Janssens, S. D. et al. Evidence for phase separation of ethanol-water mixtures at the hydrogen terminated nanocrystalline diamond surface. J. Chem. Phys. 137, 044702 (2012).

29. Dankerl, M., Hauf, M. V., Stutzmann, M. \& Garrido, J. a. Diamond solution-gated field effect transistors: Properties and bioelectronic applications. Phys. Status Solidi 209, 1631-1642 (2012).

30. Vl, Z., Petrák, V., Frank, O. \& Kavan, L. Electrochemical impedance spectroscopy of polycrystalline boron doped diamond layers with hydrogen and oxygen terminated surface. 55, 70-76 (2015).

31. Katz, E. \& Willner, I. Probing biomolecular interactions at conductive and semiconductive surfaces by impedance spectroscopy: Routes to impedimetric immunosensors, DNA-sensors, and enzyme biosensors. Electroanalysis 15, 913-947 (2003). 